\title{
Breast Auto-Augmentation: A Versatile Method of Breast Rehabilitation-A Retrospective Series of 107 Procedures
}

\author{
Laurence Kirwan, Umar Wazir, Kefah Mokbel \\ Department of Aesthetic and Reconstructive Breast Surgery, London Breast Institute, London, UK
}

Background Breast auto-augmentation (BAA) using an inferior pedicle dermoglandular flap aims to redistribute the breast tissue in order to increase the fullness in the upper pole and enhance the central projection of the breast at the time of mastopexy in women who want to avoid implants. The procedure achieves mastopexy and an increase in breast volume.

Methods Between 2003 and 2014, 107 BAA procedures were performed in 53 patients (51 bilateral, 2 unilateral and 3 reoperations) with primary or secondary ptosis of the breast associated with loss of fullness in the upper pole $(n=45)$ or undergoing explantation combined with capsulectomy $(n=8)$. Six patients $(11.3 \%)$ had prior mastopexy and $2(3.7 \%)$ patients had prior reduction mammoplasty. The mean patients' age was 41 years (range, 19-66 years). All patients had preoperative and postoperative photographs and careful preoperative markings. Follow-up ranged from 6 months to 9 years (mean, 6.6 months).

Results The range of elevation of the nipple was from 6 to $12 \mathrm{~cm}$ (mean, $8 \mathrm{~cm}$ ). The wounds healed completely with no complications in $50(94.3 \%)$ patients. Three patients had complications including $2(3.7 \%)$ hematomas and $1(1.9 \%)$ partial necrosis of the nipple-areola complex. Three (5.7\%) patients were dissatisfied with the level of mastopexy achieved underwent a further procedure. No patient complained of scar hypertrophy.

Conclusions BAA is a versatile technique for women with small breasts associated with primary or secondary ptosis. It is also an effective technique for the salvage of breasts after capsulectomy and explantation.

Keywords Breast / Mammaplasty / Surgical flaps
Correspondence: Kefah Mokbel The London Breast Institute, Princess Grace Hospital, 42-52 Nottingham Place, London W1U 5NY, UK Tel: +44-(0)20-7908-2040 Fax: +44-(0)207-908-2275 E-mail: kefahmokbel@hotmail.com

No potential conflict of interest relevant to this article was reported.

Received: 8 Feb 2015 • Revised: 1 Apr 2015 • Accepted: 13 Apr 2015

pISSN: 2234-6163 • elSSN: 2234-6171 • http://dx.doi.org/10.5999/aps.2015.42.4.438• Arch Plast Surg 2015;42:438-445

\section{INTRODUCTION}

Breast augmentation as a cosmetic procedure or as a part of oncoplastic reconstruction is an increasingly common procedure in which various techniques and methods could be employed. This may be performed by use of autologous grafts in the form of regional pedicled flaps (e.g., latissimus dorsi) or by using free flaps from the abdominal or gluteal regions. More commonly, silicone implants are employed to achieve augmentation [1]. Both techniques have their inherent limitation. Briefly, whilst flaps have the advantage of the use of the patient's own tissue, donor site morbidity can be considerable. In contrast, whilst the state of a donor site is not an issue with implants, their use is marred by limited life-span, migration and capsule formation re- 
sulting in distortion [2]. The techniques have matured considerably with use, with a resultant improvement in outcomes. However, a niche remains for the use of less onerous procedures. In this article, we shall share our experience with a technique in which the appearance of augmentation could be achieved in select patients by correction of breast ptosis.

The senior author's 2007 article [3] based on an earlier presentation 'Lollipop Mastopexy' [4] described a modification of the technique of Botti [5] and Graf et al. [6] using an inferior parenchymal pedicle transposed superiorly to the anterior face of the pectoralis major. This was combined with a vertical mastopexy [7] and a peri-areolar purse-string suture $[8,9]$. The pursestring suture was used to reduce the peri-areolar diameter, limit the length of the vertical limb and stabilize the peri-areolar closure. Furthermore, the peri-areolar purse-string reduced tension and risk of vascular compromise of the nipple areolar complex (NAC). The technique was named 'Breast Auto-augmentation' (BAA) as used in subsequent papers [10,11]. In the original paper [3] the BAA technique was used in a case of explantation. Gurunluoglu et al. [12] mistakenly credited the first use of combining BAA with explantation to Honig et al. [11].

This paper retrospectively reviews 53 patients, 56 cases of BAA, 107 individual breast surgeries in all, performed by the senior author from July 2002 to February 2014. The technique has proved to be a reliable, durable and versatile method for cases of primary ptosis and ptosis following postpartum and post-lactation involution with resultant loss of fullness of the superior pole. In a subset of cases in this series, BAA was performed in conjunction with explantation and capsulectomy as salvage procedure. These procedures could be termed as a 'Salvage Auto-augmentation' (SAA) to distinguish them from BAA performed primarily. In addition, BAA has been used in this series after prior breast mastopexy and in cases of inferior and superior pedicle breast reduction. Furthermore, BAA represents a valid alternative to the use of implants, with or without mastopexy in patients with sufficient parenchyma in the lower pole who do not desire an absolute increase in breast volume.

\section{METHODS}

A retrospective chart review was conducted of 53 consecutive patients three of whom had repeat surgical procedures. Therefore 107 breasts underwent breast-auto-augmentation, 51 bilateral, five unilateral, in 53 patients. Patients were aged 19 to 66 years (mean, 41 years). BAA was performed for correction of primary ptosis, post-partum and post lactation ptosis with loss of upper pole fullness as well as for breast salvage after capsulectomy and explantation (SAA). These latter SAA cases were for complications such as capsular contracture, ptosis, implant malposition, undesirable size and desire to remove implants, with or without prior attempted corrective surgery.

\section{Preoperative photographs}

It is recommended that in addition to the usual series of frontal and oblique photographs, a right and left lateral view, with the arms at the side, should be a standard part of the preoperative photographic series for all breast cases. This allows for a more accurate assessment of the level of the NAC and the parenchy$\mathrm{ma}$ in their relation to the chest wall thus allowing for a better evaluation of surgical results. In addition, photographs should also be taken in the frontal pose with hands on the hips or at the sides as well as on top of the head to show any vertical and inframammary scars. This view should be also repeated in both oblique positions with the hands behind the back and then on top of the head. Furthermore, a supplemental view may be taken with the patient holding the breasts up to show the full extent of any infra-mammary scar.

\section{Preoperative marking}

The patient is marked in the standing position with the arms at the side to mark the new nipple location and then, following this, with the hands on the hips as well as on top of the head to complete the inferior marking. The new nipple position is at the level of the infra-mammary crease as determined with a gynecological caliper. One end of the caliper is pressed upwards against the infra-mammary crease to determine its ideal level then transposing the same limb to the front of the breast. The other end of the caliper is meanwhile placed in the supra-sternal notch. The cephalic limit of the neo-areola is marked $2 \mathrm{~cm}$ above the new nipple location. Care should be taken to ensure that the new cephalic border of the areola is identical on the contra-lateral breast, again using the calipers. The superior level of the areola is also checked manually by grasping the areola at 3 and 6 o'clock; placing cephalic tension on the areola until the infra-areolar skin is taut. No reference is made to mid-humeral length as previously described $[3,12]$. The amount of nipple elevation varied from less than 1 to $12 \mathrm{~cm}$.

An $8 \mathrm{~cm}$ or a $9 \mathrm{~cm}$ circular diameter pattern [3] is then used to mark the area of peri-areolar skin de-epithelialization (Fig. 1). The superior edge of the circle is placed at the most cephalic mark and the inferior edge normally touches the inferior border of the areola (Fig. 1). The most medial markings of the circle should be equidistant from the midline. The margins of the peri-areolar de-epithelialization region should be adjusted so as not to reduce the intended size of the new areola. If the nipple level is greater than $12 \mathrm{~cm}$ from the superior point of de-epithelialization then 


\section{Fig. 1. Preoperative markings for breast auto-augmentation}

The patient is marked in the standing position with the arms at the side to mark the new nipple location and then, following this, with the hands on the hips as well as on top of the head to complete the inferior markings. The new nipple position is at the level of the infra-mammary crease. The cephalic limit of the neo-areola is marked $2 \mathrm{~cm}$ above the new nipple location.

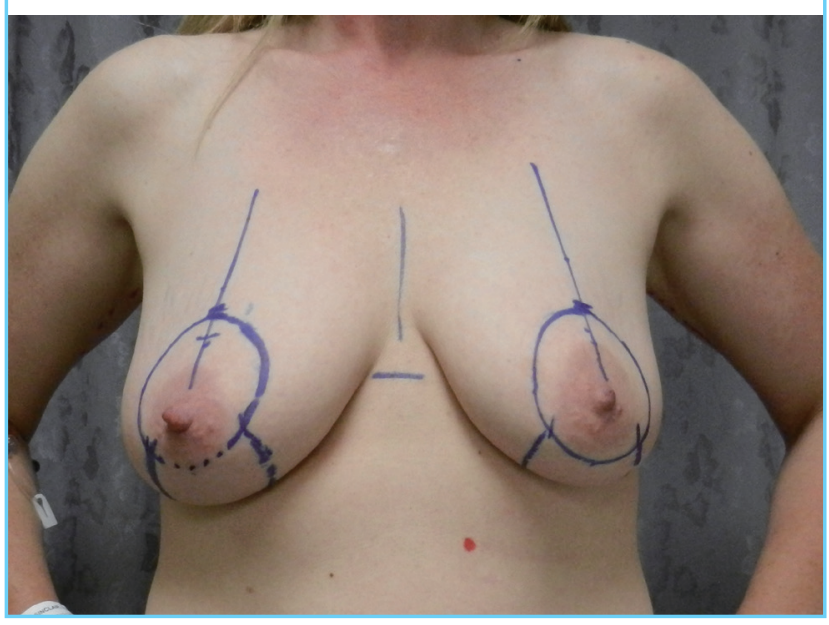

the technique of superior pedicle NAC transposition should be reconsidered in favor of an inferior pedicle technique. In the case of a longer vertical correction (greater than $9 \mathrm{~cm}$ ) the pattern is converted into a lenticular one bordering the areola margins in order to reduce the risk of horizontal skin shortage. It should be noted that the vertical distance between the cephalic and caudal limits of the peri-areolar de-epithelialization is significantly shortened when the patient is supine on the operating table and that the superior pedicle is therefore more manageable. If further excision of the peri-areolar skin is required, then this can be determined with tailor tacking at the time of closure. The medial and lateral vertical limbs of the inferior pedicle, that circumscribe the parenchymal pedicle, that is to be used for auto-augmentation, are then determined. The breast is rotated in clockwise and counter-clockwise directions until the medial and lateral limbs are in the midline; when moderate tension is placed on the breast in this fashion. These vertical limbs are continued down to the infra-mammary crease, not above the crease as previously described [3]. Further visualization can be obtained by having the patients put their hands on top of their head. At the cephalic extent of the medial and lateral limbs the line is curved towards the midline of the breast for about $5 \mathrm{~mm}$ in order to limit tension in this area. There is a minimum of $6 \mathrm{~cm}$ distance between the lateral and medial limbs at their cephalic extent. Rarely, tailor tacking is performed before de-epithelialization of the vertical ellipse. Tailor tacking is usually reserved until the time of final closure mainly to evaluate the necessity for further skin excision in the vertical plane. Tailor tacking is rarely performed in the peri-areolar region. If at the time of surgery, one areola appears significantly higher than the other, additional skin is excised superiorly to make them symmetrical.

\section{Surgical technique}

The areola is circumscribed with a $42 \mathrm{~mm}$ areola marker. The areola is stretched to the point where the marker can be applied symmetrically with the nipple at its center. A 45-mm marker was rarely used in this series since it would result in an areola that is aesthetically over-sized and usually bulges in an unsightly fashion. A smaller $38 \mathrm{~mm}$ pattern may result in asymmetrical excision of the areolar and may look disproportionately small in the case of a large nipple. De-epithelialization is performed (Fig. $2 \mathrm{~A})$. The inferior pole of the vertical limb is undermined at the sub-dermal level to free up the inferior parenchymal pedicle and to reduce the inferior skin redundancy (Fig. 2B). The inferior parenchymal pedicle is isolated with a medial incision at 90 degrees to the chest wall and the lateral incision is made obliquely to the chest wall so as to preserve the vascular supply (Fig. 2C). The superior incision is made obliquely to the superior chest wall overlying the pectoralis major (Fig. 2D). After isolation of the inferior pedicle and transposition super-medially, as previously described [3], the de-epithelialized skin of the peri-areolar region is incised peripherally from 4 o'clock to 8 o'clock, only. The NAC is fixed superiorly in the midline at the 12 oclock position with a 4-0 monofilament glycomer suture (Fig. 3A). The superior poles of the vertical limbs are apposed with a 3-0-monofilament polyglyconate suture to which a hemostat is applied (Fig. 3B). Cephalic tension is applied to this suture whilst the remainder of the inferior limb is closed with deep dermal sutures of 3-0 monofilament polyglyconate. If indicated, tailor tacking (Fig. 3C) is performed at this stage prior to final closure (Fig. 3D). Further skin and parenchyma is excised after tailor tacking, as required. No parenchymal sutures are placed and no sutures are used to tack the inferior flaps to the chest wall. A suction drain is placed inferiorly, exiting the wound just superior to the inferior pole of the vertical closure. This is removed the following day, coincident with the dressing change.

The peri-areolar closure consists of eight evenly spaced 4-0 monofilament glycomer deep dermal sutures followed by placement of a dermal 3-0 polyester suture on a SH taper needle, soaked in Betadine (Purdue Fredrick, Norwalk, CT, USA), with the knot buried. Final closure of the peri-areolar incision is done with a subcuticular suture of 4-0 monofilament glycomer suture. The vertical limb is closed with interrupted 4-0 monofilament glycomer suture and a subcuticular closure using the same suture. If necessary, inferior skin redundancy is converted into a 'J' (right breast), an 'L' (left breast) pattern, or a 'T' pattern. No 


\section{Fig. 2. Preparation of the inferior parenchymal flap}

The areola is circumscribed with a $42 \mathrm{~mm}$ areola marker. De-epithelialization is performed. The inferior pole of the vertical limb is undermined at the sub-dermal level to free up the inferior parenchymal pedicle and to reduce the inferior skin redundancy. The inferior parenchymal pedicle is isolated with a medial incision at 90 degrees to the chest wall and the lateral incision is made obliquely to the chest wall to preserve the vascular supply. (A) De-epithelialisation of the pedicle. (B) Undermining the inferior pole of the pedicle. (C) Medial and lateral incisions. (D) Superior incision.


attention is given to the length of the inferior closure since the new infra-mammary crease level is determined independently by the closure of the medial and lateral limbs. The vertical limb may therefore extend onto the anterior face of the abdomen and this is not considered to be a problem, especially if there is an oblique component to this part of the scar. Surgery takes approximately two hours.

\section{Salvage auto-augmentation}

In the case of explantation, the implant may be approached medially or laterally, whichever seems most convenient. In the case of Baker Grade III and IV contracture, a complete capsulectomy is performed. In Grade II and III cases, a portion of the capsule is removed; usually the superior half in sub-glandular cases and the anterior-superior or lateral part alone, in sub-muscular cases. No suture plication of the remaining capsule is performed. Suction drains are placed and brought out through the incision.

\section{RESULTS}

Fifty-three patients (a total of 107 breasts) have been treated with this technique in the past 11.5 years. Follow-up ranged from 6 months to 9 years (mean, 6.6 months). The age range was 19 to 66 years (mean age, 41 years). Five patients had a unilateral BAA (9\%) combined with another procedure on the opposite side (three reduction/one peri-areolar mastopexy/one augmenta- 


\section{Fig. 3. Preparation of the NAC}

The nipple areolar complex (NAC) is fixed superiorly in the midline at the 12 o'clock position with a 4-0 monofilament glycomer suture. The superior poles of the vertical limbs are apposed with a 3-0-monofilament polyglyconate suture to which a hemostat is applied. Cephalic tension is applied to this suture whilst the remainder of the inferior limb is closed with deep dermal sutures of 3-0 monofilament polyglyconate. If indicated, tailor tacking is performed at this stage prior to final closure. Fixation of the superior pole of the NAC. (B) Apposition of the superior poles of the vertical limbs. (C) Tailor tacking. (D) Closure and end result.
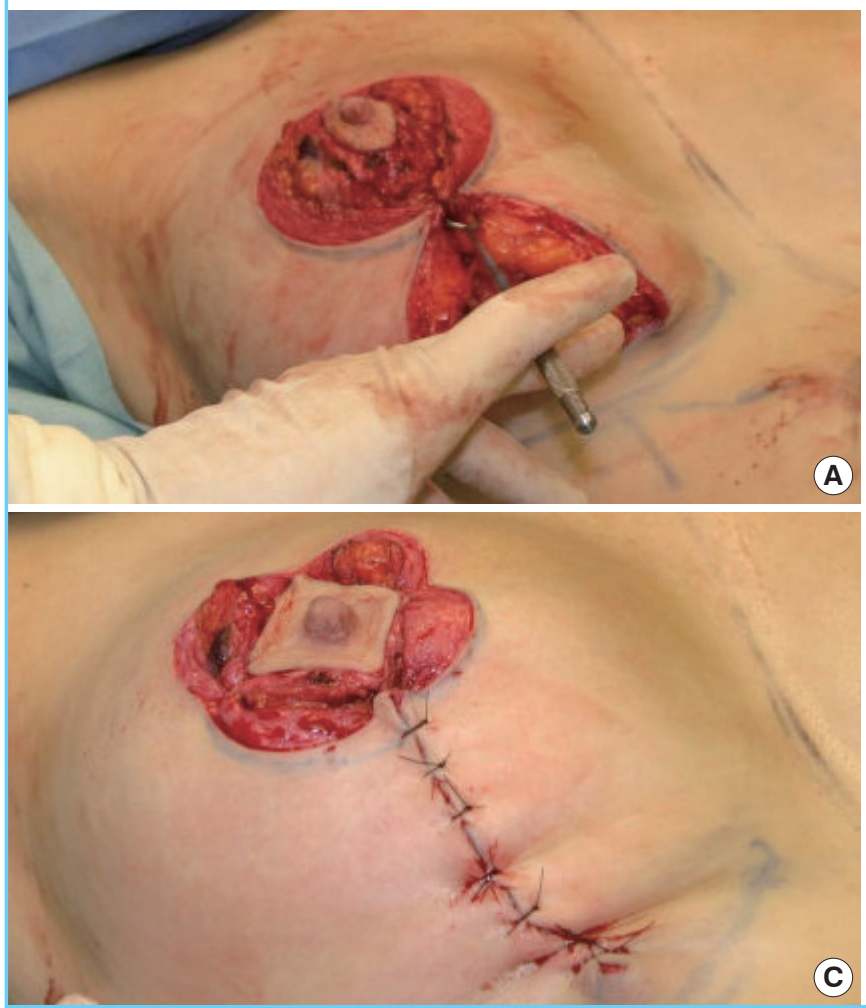

tion with a peri-areolar mastopexy). One patient had a unilateral partial reduction of an inferior pedicle.

The postoperative outcomes were assessed and recorded with photograph taken in outpatient clinics. The patients were followed-up in outpatients at one week, one month, three months, six months and one year postoperatively, with further follow-up arranged as needed.

Objectively, the range of elevation of the nipple achieved was from 6 to $12 \mathrm{~cm}$ with a mean of $8 \mathrm{~cm}$. No patient complained of a hypertrophic scar.

One patient lost $20 \mathrm{~kg}$ after surgery and subsequently had a submuscular breast augmentation.

\section{SAAcases}

Eight patients (15\%) had SAA. Four had sub-muscular and four pre-muscular implants. Three SAA patients had ruptured implants, two bilateral, one unilateral. In all, five patients had total capsulectomies; one with ruptured sub-muscular implants and bilateral Baker Grade IV capsular contracture and one with ruptured pre-muscular implants and bilateral Baker Grade III capsular contracture. Of the three other total caspulectomy cases
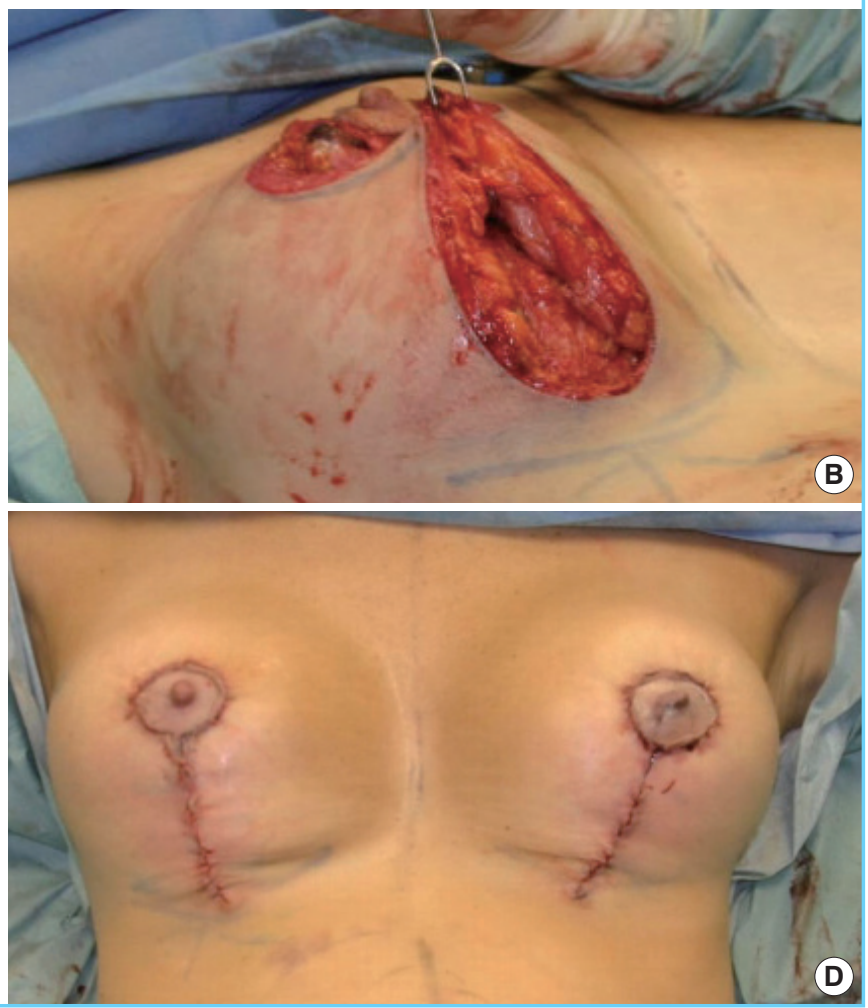

three were sub-muscular and two pre-muscular. The remaining three patients had a partial capsulectomy (one sub-muscular and two pre-muscular). Two SAA patients with sub-muscular implants presented with a "Snoopy" [13] deformity and two patients with sub-glandular implants complained of oversized ptotic breasts. Two cases of SAA with pre-muscular implants (one partial, one complete capsulectomy) had a modification of the surgical technique with preservation of the superior attachment of the inferior pedicle to the NAC and in-folding of the central pedicle.

\section{Prior mastopexy/reduction}

Six patients had prior bilateral mastopexies, two vertical superior pedicles, two vertical bucket-handle pedicles, two simultaneous areolar mastopexy breast augmentations (SAMBA) [14]. Two patients had prior breast reductions. One patient had an inferior pedicle, Wise pattern reduction, the other, a superior pedicle vertical mastopexy.

\section{Reoperations}

Three patients required a second bilateral BAA to correct persis- 


\section{Fig. 4. Case 1 (breast auto-augmentation)}

This patient is a 45 year-old white female with para 1, with bilateral ptosis Stage C. She underwent breast auto-augmentation with a $6 \mathrm{~cm}$ nipple areolar complex elevation. Front view, preoperative. (B) Front view, postoperative. (C) Right oblique view, preoperative. (D) Right oblique view, postoperative.
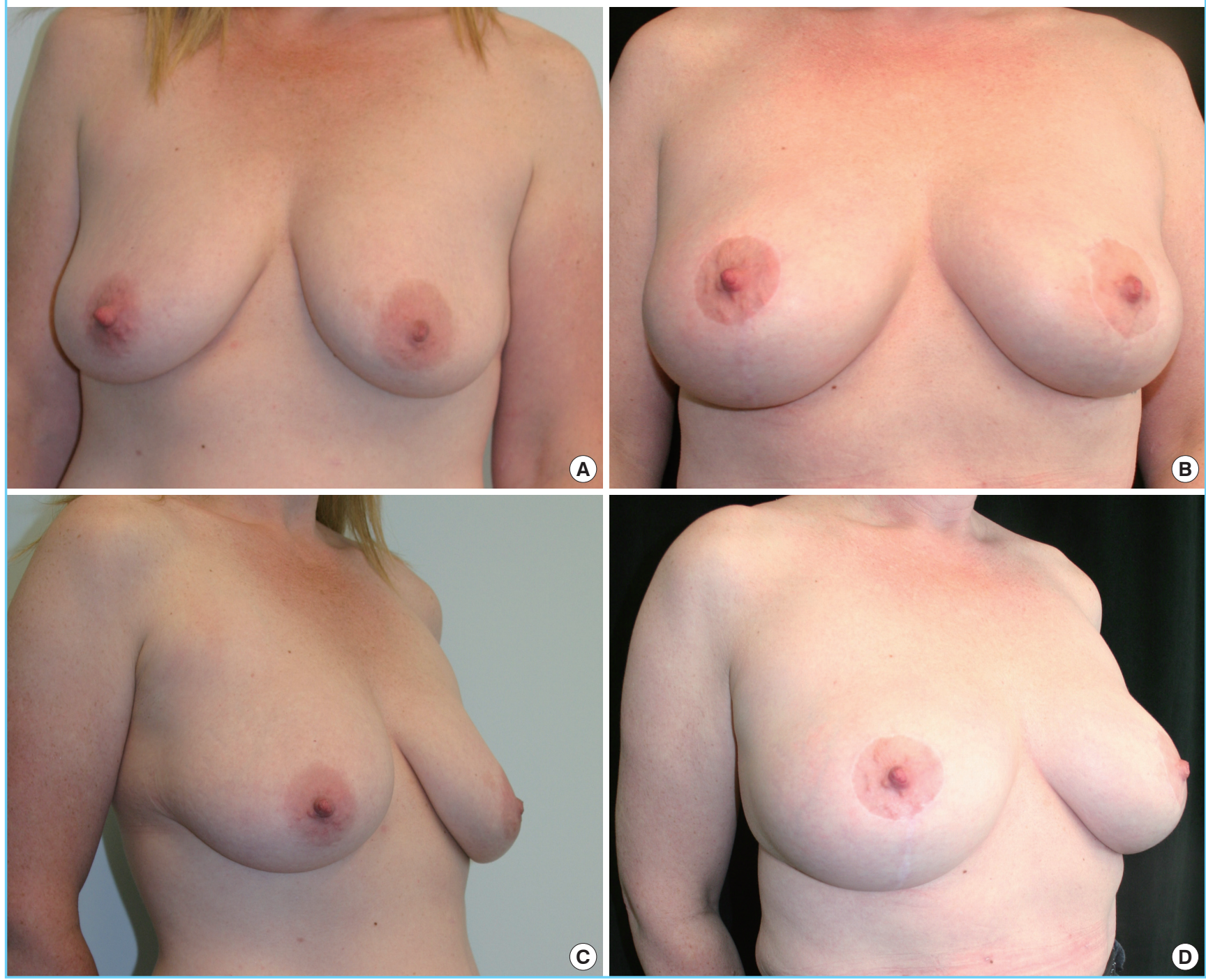

tent ptosis (5.7\%). All such secondary patients had surgery without complication and occurred early in the series (2003, 2005. 2008).

\section{Other concurrent procedures}

Eight patients had additional non-breast procedures simultaneous with the BAA. One patient had a coincidental modified brachioplasty, mini-abdominoplasty and flank lift. Six patients had coincidental liposuction and one patient had an upper and lower blepharoplasty.

\section{Complications}

Three patients had secondary mastopexy procedures because of an inadequate primary mastopexy (5.7\%). Two patients had a unilateral hematoma (3.7\%) and one of whom had a unilateral partial NAC necrosis on the left side that was satisfactorily resolved with a debridement and primary closure 25 days later. This complication occurred in 2006 and there have been no further instances since. A further patient had vascular compromise without tissue loss which resulted in partial loss of pigmentation of the areola. The total incidence of vascular complications was $2.7 \%$.

\section{Representative cases}

\section{Case 1 (Fig. 4): BAA, $6 \mathrm{~cm}$ NAC elevation}

A 45-year-old white female, Para 1, with bilateral ptosis Stage C [15]. Complaining of loss of upper pole fullness and breast ptosis. Postoperatively the patient had a hematoma of the right breast 


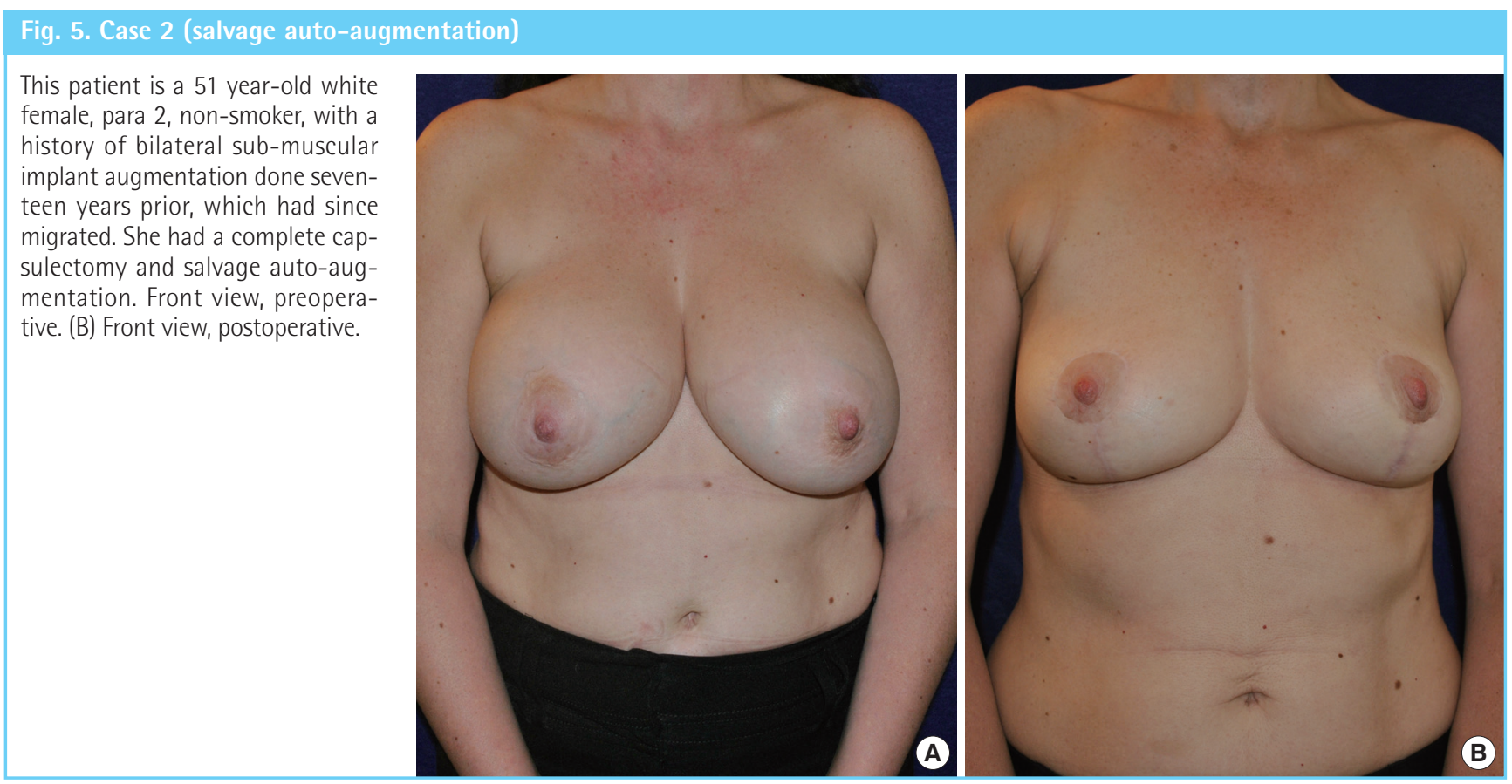

evacuated day of surgery which healed uneventfully. She had liposuction of the arms and abdomen (total fat volume $300 \mathrm{cc}$ ) and a scar revision of a midline lower infra-umbilical scar at the same time as the primary surgery. Thirty-one month follow-up.

\section{Case 2 (Fig. 5): SAA}

A 51-year-old white female, Para 2, non-smoker, with history of bilateral sub-muscular augmentation, seventeen years prior. Complete capsulectomy and auto-augmentation. Patient stated that she had lost nipple sensation with the initial breast augmentation and had return of sensation of both nipples after bilateral SAA. Fourteen month follow-up.

\section{DISCUSSION}

The technique has been used after prior breast mastopexy as well in cases of inferior and superior pedicle breast reduction. BAA is an alternative to the use of implants, with or without mastopexy provided the patient has sufficient parenchyma in the lower pole and does not desire an absolute increase in breast volume. This choice is also patient dependent.

BAA [3] corrects the empty upper pole as well as the sagging of the primary ptotic, post-partum and post-lactation breast resulting in the appearance of an augmented breast and a scar in the configuration of a 'lollipop' [4]. BAA is also a versatile method of salvaging the explanted patient. Indications for removal of implants are: desire not to have implants, appearance, size reduction, capsular contracture, leaking implants and breast implant malposition in a sub-muscular or sub-glandular plane. Preoperative marking is simple and can be modified at the time of surgery. The use of a permanent purse-string suture combined with a peri-areolar mastopexy $[3,8,9]$, stabilizes the shape of the areola. This reduces the possibility of areola 'creep' which results in a vertical - elliptical, instead of a circular areolar shape. In addition, the circum-areolar purse-string suture prevents tension and the risk of compromised vascularity of the areola. The technique is different from the BAA technique of Gurunluoglu et al. [12] that utilizes a standard Wise pattern skin excision and closure.

Minimal mobilization and undermining of the NAC is performed so as to limit the risk of ischemia. Keyhole patterns are avoided, since they narrow the superior pedicle, in favor of the circular pattern described [3]. Dermal release of the superior pedicle is limited from 4 o'clock to 8 o'clock only. The remainder of the dermis is left untouched, contrary to the technique of Hammond [16] and Hammond et al. [17]. In addition, the diameter of the peri-areolar skin excision along with the pursestring closure, results in a reduction of the length of the vertical limb [3]. How much, depends on the size of the circle as well as the original diameter of the areola.

BAA is indicated in cases of breast ptosis Stage A to F [15] and is an alternative technique to other methods such as the use of acellular dermal graft with re-augmentation and T-shaped mastopexy in cases of the multiply operated breast augmentation [18] as well as fat injection [19]. BAA is not indicated in cases in which there is inadequate breast tissue in the inferior pole of the breast to utilize for internal auto-augmentation. Alternatives 
techniques such as fat injection have the potential risks of secondary surgery due to inadequate augmentation, absorption of graft, nodules and fat necrosis $[19,20]$. Fat injection may also have the not insignificant downside of pre-surgical expansion with the Brava device [19].

The inferior parenchymal pedicle in the auto-augmentation, may be used in whole or in part, depending on the final desired size of the reconstruction. The vertical limb of the skin closure is continued onto the chest wall and rarely converted into a ' $\mathrm{T}$ ' shape. A slight obliquity is recommended to avoid a scar contracture across the infra-mammary crease. The continuation of the vertical limb may be as a slight obliquity (as above) or as a 'J' or ' $\mathrm{L}$ ' shape in the case of the right and left breast, respectively. Rarely, the incision will be converted into a ' $\mathrm{T}$ ' configuration at the infra-mammary crease. The length of the infra-areolar closure does not determine the distance between the inferior areola margin and the infra-mammary crease. As the new breast is reconstituted and the vertical limb is closed a new infra mammary crease defines itself, based on the residual inferior hemispheric breast tissue.

BAA remains a versatile durable technique for primary breast ptosis, as well as in cases of post-partum and post-lactation ptosis and involution. It is a useful and effective technique for salvage of breasts after capsulectomy and explantation. It is a much less complicated and less costly alternative than the use of acellular dermal graft with implant augmentation and fat transfer. In addition, the authors' technique is limited to a 'lollipop' scar in the original spirit and mode of the vertical breast technique, rather than sacrificing the brevity of the scar for a Wise pattern. The procedure is easy to plan, surgery time is short and complications are acceptably low.

\section{REFERENCES}

1. Hidalgo DA, Spector JA. Breast augmentation. Plast Reconstr Surg 2014;133:567e-583e.

2. Mlodinow AS, Ver Halen JP, Lim S, et al. Predictors of readmission after breast reconstruction: a multi-institutional analysis of 5012 patients. Ann Plast Surg 2013;71:335-41.

3. Kirwan L. Breast autoaugmentation. Can J Plast Surg 2007; 15:73-6.

4. Kirwan L. FP315: lollipop mastopexy, combined periareolar and vertical mastopexy. ANZ J Surg 2003;73 (Supplement s2):A224.
5. Botti G. Vertical scar mammaplasty: stable padding of the superior pole by means of posteriorly based pedicle autoprosthesis. Aesthet Surg J 1999; 19:116-23.

6. Graf R, Reis de Araujo LR, Rippel R, et al. Reduction mammaplasty and mastopexy using the vertical scar and thoracic wall flap technique. Aesthetic Plast Surg 2003;27:6-12.

7. Lassus C. A 30-year experience with vertical mammaplasty. Plast Reconstr Surg 1996;97:373-80.

8. Benelli L. A new periareolar mammaplasty: the "round block" technique. Aesthetic Plast Surg 1990;14:93-100.

9. Kalenderof V, Tumerdem B, Aydin H, et al. Modification of vertical scar in vertical mammaplasty technique. Aesthetic Plast Surg 2001;25:40-2.

10. Honig JF, Frey HP, Hasse FM, et al. Inferior pedicle autoaugmentation mastopexy after breast implant removal. Aesthetic Plast Surg 2010;34:447-54.

11. Honig JF, Frey HP, Hasse FM, et al. Autoaugmentation mastopexy with an inferior-based pedicle. Aesthetic Plast Surg 2009;33:302-7.

12. Gurunluoglu R, Sacak B, Arton J. Outcomes analysis of patients undergoing autoaugmentation after breast implant removal. Plast Reconstr Surg 2013;132:304-15.

13. Schulz CM. Around the world in 50 years: Charlie Brown's anniversary celebration. Kansas: Andrews McMeel Publishing; 1994.

14. Kirwan L. Augmentation of the ptotic breast: Simultaneous periareolar mastopexy/breast augmentation. Aesthet Surg J 1999;19:34-9.

15. Kirwan L. A classification and algorithm for treatment of breast ptosis. Aesthet Surg J 2002;22:355-63.

16. Hammond DC. Short scar periareolar inferior pedicle reduction (SPAIR) mammaplasty. Plast Reconstr Surg 1999; 103:890-901.

17. Hammond DC, Hidalgo D, Slavin S, et al. Revising the unsatisfactory breast augmentation. Plast Reconstr Surg 1999; 104:277-83.

18. Maxwell GP, Gabriel A. The neopectoral pocket in revisionary breast surgery. Aesthet Surg J 2008;28:463-7.

19. Del Vecchio DA, Bucky LP. Breast augmentation using preexpansion and autologous fat transplantation: a clinical radiographic study. Plast Reconstr Surg 2011;127:2441-50.

20. Mu DL, Luan J, Mu L, et al. Breast augmentation by autologous fat injection grafting: management and clinical analysis of complications. Ann Plast Surg 2009;63:124-7. 